\title{
THE INTERACTION IN VARIOUS MANIFESTATIONS (OBSERVATIONS ON LILLIE'S NERVE MODEL)
}

\author{
PART II. THE EFFECTS ON THE DISTANCE \\ TRAVELLED AND THE REFRACTORY PERIOD
}

\author{
KAZUMI YAMAGIWA * \\ Department of Physiology, Tokyo Medico-Dental College, Tokyo
}

There were described, in Part I (1) of this article, some detailed studies on the accelerating interaction between two Lillie's nerve models lying in parallel and close to each other. The marked accelerating effect, a sort of after-effect, of one model activated upon the conduction velocity of the other, suggested to the author that the action would manifest itself as well in some other things as in the conduction velocity, and this suggestion made him pay attention to the possible elffects on the distance travelled and on the duration of the refractory period of the model affected.

The result were positive and interesting, which will be reported here briefly in the following pages. The apparatus and other are all the same as before (refer to Part I (1)).

THE EFFECT ON THE DISTANCE TRAVELLED

Two cores, I and II, were laid in parallel as usual, $5 \mathrm{~mm}$. apart from each other. What was done actually was the measurement of the distances travelled by waves in the core II by means of a scale attached to one side of the groove, the container for the cores. The measurement was done twice successively, or many times alternatively, with and without the activation of the core I prior to that of the core II. The object was to estimate thus the influence of the activation of the core I upon the conductivity of the core II.

The cores were made, on purpose, from two different samples. The core I was about $1 \mathrm{~mm}$. thick and had a refractory period of shorter than $30^{\prime \prime}$, while the core II was about $0.5 \mathrm{~mm}$. thick and of a refractory period longer than $2^{\prime}$. By "refractory period" is meant here the least interval of two successive stimuli which is just effective to make the wave conduct along the whole length, and not that for the local response. The latter is of course very much shorter, because the distance travelled depends on the stimulus interval, as will soon be described. The reason why the core I possessing a very short refractory period was chosen as the accelerator, is that its activation wave can sweep along the whole length even in the case of very short immersion times and can thus affect the whole length of the core II uniformly, so that it is convenient for examining the cases of short immersion times. Care was taken to maintain three important

\footnotetext{
1 山極一三

Received for Publication April 7, 1950.
} 
conditions, namely, 1) the constancy of the immersion time of the core II, 2) the constancy of the interval between $R_{1}$ and $R_{2}$, the stimuli applied to the cores I and II respectively, and 3) the avoiding of the direct effect of $R_{1}$ on the core II. The former two were realised by fixing the interval between two successive $R_{2}$ S and by applying $R_{1}$ a short definite time ( $5^{\prime \prime}$ in most of the cases) before every predetermined moment of $R_{2}$. For the third condition to be realised, the stimulus $R_{1}$ was applied to the up-right part of the core I, which had been passivated previously with other parts and was exposed to the air just before the moment of stimulation. Another simple method adopted at times is shown in Fig. 1, which will need no explanation. The result obtained was always unique and beautiful, as shown in Table 1 and Fig. 2 .

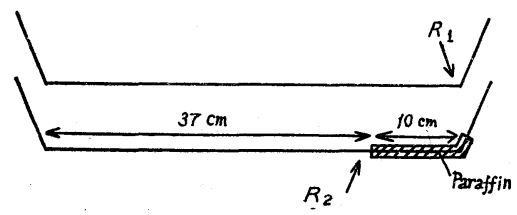

Fig. 1

The distance travelled in the case of $2^{\prime} 15^{\prime \prime}$ immersion time (the stimulus was single, that is, $R_{2}$ only) and the same for $1^{\prime} 30^{\prime \prime}$ immersion time (the stimulus was double, that is, $R_{1} \rightarrow R_{2}$ ) were both taken as $37 \mathrm{~cm}$. in Fig. 2, but " $37 \mathrm{~cm}$." means here simply the whole length of the core and not the real length which was to be travelled in case the core had been sufficiently long. The real lengths must have been much longer, although we don't know how much, hence the points which represent those, should be situated much higher than those plotted in the figure. Anyway, both curves go upwards slowly to begin with and acutely afterwards, to become almost perpendicular at a certain immersion time.

Now, the two curves in Fig. 2, taken together, clearly indicate the augmenting effect of the activation wave of the core I upon the distance travelled in the core II, the intensity of which increases with longer immersion time. On the other hand, the shape of either of the curves, taken separately, indicates most clearly (although it has no direct connection with the present problem) that the conduction distance is

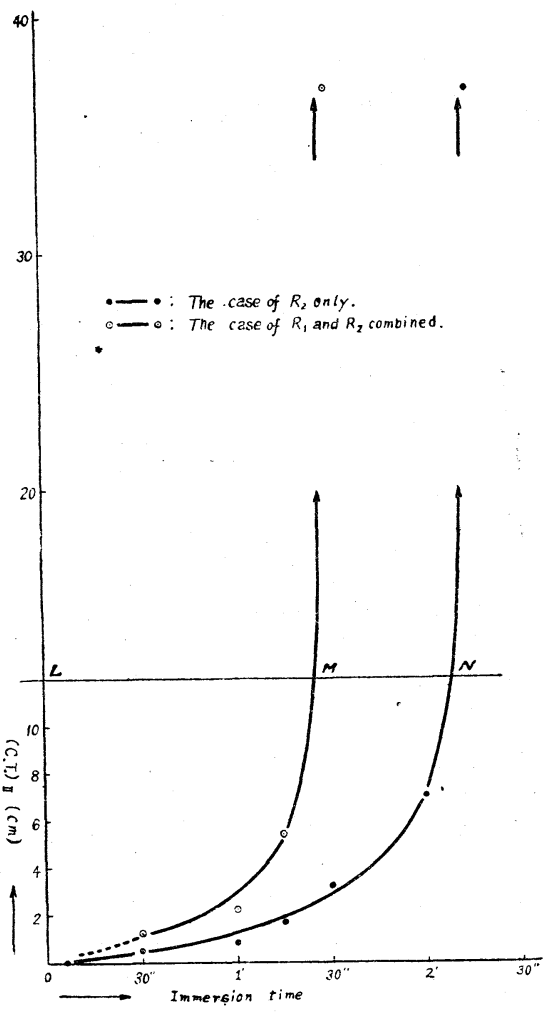

Fig. 2 
Table 1

\begin{tabular}{|c|c|c|c|c|}
\hline $\begin{array}{l}\text { Imm. } \\
\text { time }\end{array}$ & Time & Stimulus & $\underset{(\mathrm{cm} .)}{(\text { Distance trav. })} \mathrm{II}$ & Remark \\
\hline & $\begin{array}{c}\text { 3h. } 31^{\prime 1)} \\
37^{\prime} \\
40^{\prime}\end{array}$ & $R_{1},{ }_{\| 2)}^{\left.R_{2}^{2}\right)}$ & $\overline{-}$ & $\begin{array}{l}\text { 1) The cores settled. } \\
\text { 2) Smoothing stim. }\end{array}$ \\
\hline $1^{\prime}$ & $\begin{array}{l}41^{\prime} \\
42^{\prime} \\
43^{\prime} \\
44^{\prime}\end{array}$ & $\begin{array}{l}R_{2} \\
R_{1} \rightarrow R_{2} \\
R_{2} \\
R_{1} \rightarrow R_{2}\end{array}$ & $\begin{array}{l}0.7 \mathrm{~cm} . \\
2.5 \\
1 \\
2\end{array}$ & \\
\hline $30 "$ & $\begin{array}{l}44^{\prime} 30^{\prime \prime} \\
45^{\prime} \\
45^{\prime} 30^{\prime \prime} \\
46^{\prime}\end{array}$ & $\begin{array}{l}R_{2} \\
R_{1} \rightarrow R_{2} \\
R_{2} \\
R_{1} \rightarrow R_{2}\end{array}$ & $\begin{array}{l}0.5 \\
1.5 \\
0.5 \\
1\end{array}$ & - \\
\hline $1^{\prime} 30^{\prime \prime}$ & $\begin{array}{l}48^{\prime} \\
49^{\prime} 30^{\prime \prime} \\
51^{\prime} \\
52^{\prime} 30^{\prime \prime} \\
54^{\prime}\end{array}$ & $\begin{array}{l}R_{1}, R_{2}{ }^{3)} \\
R_{2} \\
R_{1} \rightarrow R_{2} \\
R_{2} \\
R_{1} \rightarrow R_{2}\end{array}$ & $\begin{array}{l}\frac{-}{3} \\
\text { The whole length } \\
3.3 \\
\text { The whole length }\end{array}$ & 3) Smoothing stim. \\
\hline $1^{\prime} 15^{\prime \prime}$ & $\begin{array}{l}55^{\prime} 15^{\prime \prime} \\
56^{\prime} 30^{\prime \prime} \\
57^{\prime} 45^{\prime \prime} \\
59^{\prime}\end{array}$ & $\begin{array}{l}R_{2} \\
R_{1} \rightarrow R_{2} \\
R_{2} \\
R_{1} \rightarrow R_{2} \\
\end{array}$ & $\begin{array}{l}1.8 \\
6.5 \\
1.5 \\
4\end{array}$ & 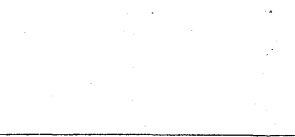 \\
\hline $2^{\prime}$ & 4h. $\begin{array}{l}1^{\prime} \\
3^{\prime} \\
5^{\prime}\end{array}$ & $\begin{array}{l}\left.R_{1}, R_{2} 4\right) \\
R_{2} \\
" \prime\end{array}$ & $\begin{array}{l}- \\
8 \\
8\end{array}$ & 4) Smoothing stim. \\
\hline $2^{\prime} 30^{\prime \prime}$ & $\begin{array}{c}7^{\prime} 30^{\prime \prime} \\
10^{\prime}\end{array}$ & $\underset{ }{R_{I I}}$ & The whole length & \\
\hline \multirow[t]{2}{*}{$2^{\prime} 15^{\prime \prime}$} & $\begin{array}{l}12^{\prime} 15^{\prime \prime} \\
14^{\prime} 30^{\prime \prime}\end{array}$ & $\underset{\prime \prime}{R_{2}}$ & " & \\
\hline & $\begin{array}{r}16^{\prime} \\
5^{\prime \prime} \\
15^{\prime \prime}\end{array}$ & $\begin{array}{l}\left.R_{1}, R_{2}{ }^{5}\right) \\
R_{2} \\
{ }^{\prime \prime}\end{array}$ & $\begin{array}{l}\overline{0} \\
0.1\end{array}$ & 5) Smocthing stim. \\
\hline
\end{tabular}

increased progressively with increased immersion time, to get finally practically infinity at a certain immersion time. This fact resembles much the phenomenon of "graded responses" of muscle fibres (Gelfan and others (2)), and suggests, it seems to me, one of the most fundamental features adherent to the mode of muscular or nervous conduction. The innermost nature of the "graded responses" is not yet quite clear, but the author's idea of "length-to-length" conduction is not incapable of making it comprehensive theoretically (Yamagiwa (3)). A theme to be attacked on this line is to examine whether the nervous impulse is always conducted along the whole length or it vanishes somewhere on the way according to the stimulative condition. If the latter is the case, we should further examine how far and with what velocity the impulse is conducted. It will be extremely hard to do so, because we shall perhaps have to perform quantitative examinations in the very vicinity of the stimulated "point." It seems, however, to be certain at least that there are graded local responses in nerve, 
too, as in muscle (Katz and others (4)).

\section{THE INFLUENCE ON THE REFRACTORY PERIOD}

\section{Examinations on uniform cores.}

When we speak of "refractory period," we think of the least interval between two successive stimuli just effective for the "local response." Usually, however, we do not think clearly of it and take conventionally a response at a distant point (in an extreme case, the muscle contraction) as an indicator.

These both coincide with each other if only the nerve fibre follows the allor-none law in the meaning that an excitation wave, once started, is always conducted away to the end of the fibre. But, as was described above, there are grades of local responses, short of propagated impulses, of nerve, hence we have to distinguish clearly the two kinds of refractory period.

In the model experiment, the refractory period for the local response was so short as 5 seconds or so. So it was impossible, in the present technique, to examine the influence on it of the activation wave of a neighbouring core, and we had to take up the refractory period for the conducted wave as the only examinable.

Now, we need not perform any new experiments for the present object, because the experimental results described in the foregoing chapter express just what we wish to know. Refer to Fig. 2, and draw an arbitrary line, $L M N$, parallel to the $x$-axis, and give attention to $M$ and $N$, the points of intersection with the curves. $L M$ and $L N$ represent the minimum immersion time necessary for the conduction as far as $L \mathrm{~cm}$. affected and unaffected by the activation of the neighbouring core respectively. The minimum immersion time is, in fact, nothing but the minimum stimulation interval, which, again, is nothing but the refractory period. The figure shows us, therefore, that the refractory period, estimated at a $L \mathrm{~cm}$. distance from the start, is shortened from $L N$ to $L M$ by the activation of the neighbouring core. The difference $M N$ gets larger with larger $L$ and attains finally a practically constant value when $L$ is taken larger than a certain limit.

\section{Examinations on partly refractory cores.}

Suppose that a part, $P Q$, of the core II in Fig. 3 is made refractory, and, under a fixed immersion time, a stimulus is given at the right end of the core. The wave will come along as usual, but it will be interrupted at the entrance, $\boldsymbol{Q}$, or somewhere in the region, $P Q$, according to whether the region is absolutely or relatively refractory. What will then occur if a stimulus $R_{1}$ is given to the core I just before the $R_{2}$-application to the core II ?

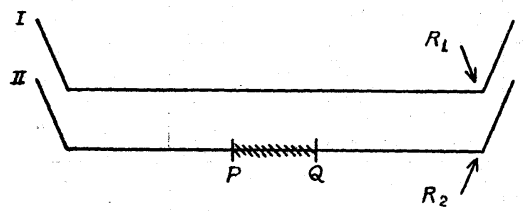


To make the part $P Q$ refractory, we activate it by a stimulus $R_{p}$ (see Fig. 4 ) a short time previous to the stimulus $R_{2}$, and to check the activation at $P$ and $Q$, we have only to make platinum-contacts at those points, holding a piece of Pt-wire in such a position as shown in Fig. 4. Care was taken thereby to keep the contact duration constant, because $\mathrm{Pt}$ exerts a strong electrotonic effect. The $\mathrm{Pt}$-wire was of course taken off as soon as the region $P Q$ was activated by the stimulus $R_{p}$.

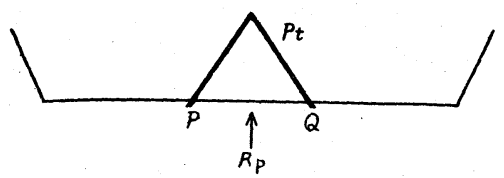

Fig. 4

The stimuli applied were $R_{p}+R_{2}$ in one case, and $R_{p}+R_{1}+R_{2}$ in the other. At a predetermined moment, they were given successively and as quick as possible (in a few seconds in all) in the order $R_{p} \rightarrow R_{2}$ and $R_{p} \rightarrow R_{1} \rightarrow R_{2}$ respectively.

The point of observation was whether the wave due to $R_{2}$ passes through the region $P Q$ or not in those two cases. The examination was repeated a great many times, varying the immersion time, on one side, and the length of the region $P Q$, on the other. The result was, briefly to say, that even in such a case as the wave is interrupted at the point $Q$ when $R_{2}$ alone is given, the wave can easily pass through the region $P Q$, if $R_{2}$ is combined with $R_{1}$.

Table 2

\begin{tabular}{|c|c|c|c|c|}
\hline Imm. & $\begin{array}{l}\text { Length } \\
\text { of } P Q\end{array}$ & Stimuli & Pass or stop at $Q$ & Remark \\
\hline \multirow{7}{*}{$2^{\prime}$} & $2 \mathrm{~cm}$. & $\begin{array}{l}R_{p}+R_{2} \\
R_{p}+R_{1}+R_{2}\end{array}$ & $\begin{array}{l}\text { Stop } \\
\text { Pass }\end{array}$ & 4 times repeated \\
\hline & $1 \mathrm{~cm}$. & $\begin{array}{l}R_{p}+R_{2} \\
R_{p}+R_{1}+R_{2}\end{array}$ & $\begin{array}{l}\text { Stop } \\
\text { Pass }\end{array}$ & $"$. \\
\hline & $0.5 \mathrm{~cm}$ & $R_{\nu}+R_{2}$ & Pass & 3 times repeated \\
\hline & $3 \mathrm{~cm}$. & $R_{p}+R_{1}+R_{2}$ & Pass & Once . \\
\hline & $4 \mathrm{~cm}$. & $" 1$ & Pass & Once \\
\hline & $5 \mathrm{~cm}$. & $"$ & Pass & Once \\
\hline & $6 \mathrm{~cm}$. & $"$ & Stop & Once \\
\hline \multirow{6}{*}{$1^{\prime}$} & $1 \mathrm{~cm}$. & $R_{p}+R_{2}$ & Stop & 3 times repeated \\
\hline & $3 \mathrm{~cm}$. & $\begin{array}{l}R_{p}+R_{2} \\
R_{p}+R_{1}+R_{2}\end{array}$ & $\begin{array}{l}\text { Stop } \\
\text { Pass }\end{array}$ & Twice repeated \\
\hline & $4 \mathrm{~cm}$. & $R_{p}+R_{1}+R_{2}$ & Pass & Once \\
\hline & $4.5 \mathrm{~cm}$. & $"$ & Pass & Once \\
\hline & $5 \mathrm{~cm}$. & $" \prime$ & $\begin{array}{l}\text { Once pass, once } \\
\text { stop at the middle }\end{array}$ & Twice repeated \\
\hline & $6 \mathrm{~cm}$. & $"$ & Stop & $" \prime$ \\
\hline
\end{tabular}


Table 2, which was made by simplifying a protocoll obtained on one day, will show it clearly.

We see from Table 2 that in the case of $R_{2}$ (alone)-application, the wave passes through the region $P Q$ only when $P Q$ is smaller than $1 \mathrm{~cm}$. whereas, if $R_{1}$ is combined with $R_{\mathbf{2}}$, then the wave can pass through even when $P Q$ is so long as $5 \mathrm{~cm}$. Here we see a clear and strong effect of the activation wave of the core I, which acts on the region $P Q$.to shorten the refractory period.

\section{Remarks :}

1) In the case of the combined stimulation $\left(R_{p}+R_{1}+R_{2}\right)$, the interval between $R_{p}$ and $R_{2}$ can be a little longer (although $2^{\prime \prime}-3^{\prime \prime}$ at most) than that in the case of $\left(R_{p}+R_{2}\right)$, during which the region $P Q$ might get recovered a little and could cause the "Pass." In order to make this clear the interval between $R_{p}$ and $R_{2}$ in a $\left(R_{p}+R_{2}\right)$-application was made so long as $10^{\prime \prime}$ for trial, but still the wave did stop at the point $Q$.

2) If the order of stimuli is changed from $R_{p} \rightarrow R_{1} \rightarrow R_{2}$ to $R_{1} \rightarrow R_{p} \rightarrow R_{2}$, the wave can never pass through the region even if $P Q$ is made $1 \mathrm{~cm}$. long.

3) An interesting fact was observed when $P Q$ was shorter than $1 \mathrm{~cm}$. and was in the relative refractory period. Namely, the wave due to $R_{2}$ proceeds on into the region $P Q$ as was expected, but at the same time, two new waves start from the point $P$ in opposite directions. One proceeds on distally, and the other back into the region $P Q$ to meet with the wave coming down from the point $Q$ and to vanish at just about the middle. This is a direct evidence of the distant effect of the activation wave, which exactly coincides with the well-known observation on "cold block" of Hodgkin (5) and with that of Tasaki (6), which demonstrates the earlier excitation of a farther Ranvier node in certain conditions.

4) Another series of measurements were done on the minimum interval $x$ between $R_{p}$ and $R_{2}$ (alone applied), and $y$ between $R_{p}$ and $R_{1}+R_{2}$ (combined), which are just necessary for the wave to pass through the region $P Q$ of a fixed length. It was found that $x$ is always unmistakably larger than $y$.

\section{SUMMARY AND CONCLUSION}

The interaction between two Lillie's nerve models, was examined from two points of view. The results obtained are as follows.

1. The conductivity-enhancing action.

An activation wave of one core acts on a neighbouring core so as to make the distance travelled by a wave in the latter more or less larger than usual. The action gets stronger with larger immersion times at which the examinations are made. It seems that there is a certain critical immersion time, at which the distance travelled tends, practicaly to say, from finite to infinity.

2. The recovery-promoting action.

a). The refractory period estimated at a distant point, taking the arrival of an activation wave there as the indicator, is shortened by an activation wave sweeping along a neighbouring core. 
b). A wave, which is incapable of passing through a refractory range of a core, becomes capable of it if helped by a previous activation of a neighbouring core.

Most of the examinations described in Parts I and II of this article were nothing but those directed on the action of an activated core upon a resting neighbour.

The actions revealed are of various appearances, but essentially they point to just one thing, that is, in one word, an "excitatory" action in the broad sense of the word. This action is unquestionably based on the activation current flowing in and out of the activated core through the resting one. The current must alter the electro-chemical state of the "membrane" of the core in some or other way, and if judged from the result, the alteration aroused must be, after all, "anodic," which is exciting in the model. How such an alteration is left behind and how it is distributed along a core after a sweep of a waye in another core, is not so simple a question to be answered exactly, but it seems theoretically not improbable; either. We have, however, a more important question, that is, whether the same action takes place in living nerves, too, or not. If it does, it may serve much, together with the simultaneous interaction (Yamagiwa (7)), in making us understand the nervous functioning as a whole.

\section{REFERENCES}

1. YAMAGIWA, K. This $J .1: 40,1950$.

2. Gelfan, H. Am. J. Physiol. 93 : 112, 1930; 96 : 16, 1931, J. Physiol. $80: 285,1934$. GelfaN, H. AND G. H. Bishop. Am. J. Physiol. $101: 678,1932 ; 103: 237,1933$.

Gelfan, H. AND R. W. Gerard. Am. J. Physiol. $95: 412$, 1930, etc.

3. YamaGIWA, K. J. Jap. Physiol. Soc. 5 : 1, 1940; Jab. Med.J. 1 : 439, 1948.

4. KATZ, B. J. Neurophysiol. 5: 169, 1942.

KUFfLER, S. W. J. Neurophysiol. 5 : 309, 1942.

PUMPheRY, R. J., O. H. SCHMitT AND J. S. Young. J. Physiol. $98: 47,1940$.

SchMitT, F. O. AND O. H. SchmitT. J. Physiol. $98: 26,1940$,

5. HodGKin, A. L. J. Physiol. 90 : 183, 1938.

6. TASAKI, I. The Physiology of nerve fibres (in Japanese). p. 126 and 142, 1944; Pflueger's Ar. Physiol. 246 : 33, 1943.

7. YAMAGIWA, K. Jap. Med. J. 1: 557, 1948.

The expenses for this work were defrayed by a grant from the National Research Council of Japan. 